\title{
INEQUIDADES SOCIALES EN ATENCIÓN MATERNA
}

Social inequity in maternal care

Flor de María Cáceres-Manrique, M.Sc.*, Gloria Molina-Marín, Ph.D.**

Recibido: marzo 29/10 - Aceptado: agosto 9/10

\section{RESUMEN}

Introducción y objetivo: el presente artículo tiene como objetivo reflexionar sobre las inequidades sociales que afectan la salud de las mujeres embarazadas. Las inequidades, definidas como desigualdades injustas y evitables, son frecuentes a pesar de los acuerdos internacionales, políticas y programas que promueven la equidad en salud. Existen enfoques teóricos y metodológicos para realizar investigación sobre inequidades sociales que ameritan ser probados en el contexto para obtener evidencia y orientar las intervenciones.

Palabras clave: desigualdades en salud, factores socioeconómicos, mujeres embarazadas, atención a la salud, accesibilidad a los servicios de salud.

\section{SUMMARY}

Introduction and objective: this article was aimed at reflecting on the social inequities affecting pregnant women's health. Inequities are defined as being unfair and avoidable disparities which are prevalent in spite of international agreements, policies and programmes promoting equity in health. Some theoretical and methodological approaches for conducting research on social

\footnotetext{
* Enfermera, Magíster en Epidemiología, Profesora Asistente, Departamento de Salud Pública, Escuela de Medicina, Universidad Industrial de Santander. Estudiante de Doctorado en Salud Pública, Universidad de Antioquia. Medellín (Colombia). Correo electrónico: fmcacer@uis.edu.co,flordemar200@yahoo.com

** Enfermera, Ph.D. in Health Services Management. Profesora Asociada, Facultad Nacional de Salud Pública, Universidad de Antioquia. Medellín (Colombia).
}

inequities deserve to be tested within the context of providing evidence and guiding interventions. Key words: inequality in health, socioeconomic factor, pregnant women, health care, health service accessibility.

\section{INTRODUCCIÓN}

Las inequidades son definidas como desigualdades evitables e injustas, ${ }^{1}$ y a su vez, inadmisibles e intolerables en una sociedad de derechos. ${ }^{2} \mathrm{Su}$ estudio es muy importante para la salud pública y ha tenido auge en el presente siglo, sobre todo en lo relacionado con la manera como coadyuvan a producir enfermedades y eventos adversos. ${ }^{3}$ Los pioneros de la investigación sobre el tema han aportado evidencias, postulados teóricos y marcos de referencia para su estudio. ${ }^{4}$

Por otra parte, el bienestar de las mujeres embarazadas es una prioridad para la salud pública, tanto que la Organización Mundial de la Salud (OMS) ha recomendado el establecimiento de programas de control prenatal (CPN) para todas las gestantes en el mundo, contando con el compromiso de cada país para ofrecer atención adecuada, oportuna y de calidad. ${ }^{5}$ El programa tiene como objetivo vigilar la evolución del embarazo, evitar la morbilidad y mortalidad materna y perinatal, y por último, preparar a las embarazadas para el parto, la maternidad y la crianza.

Sin embargo, ni la cobertura, ni la asistencia al CPN alcanzan el $100 \%$ de las gestantes ${ }^{6}$ además se presentan demoras en su inicio, ${ }^{5}$ por lo que se 
considera que existen inequidades sociales (IS) que impiden a las mujeres hacer uso a plenitud de estos beneficios. ${ }^{7}$

El presente ensayo tiene como objetivo reflexionar sobre las IS en la atención materna, para ello se iniciará con una reseña sobre la evolución del estudio de las IS y sus enfoques teóricos, los acuerdos internacionales sobre salud materna, la evidencia al respecto y, por último, las necesidades de investigación sobre las IS en la atención materna en el contexto.

\section{El estudio de las inequidades sociales en salud}

El estudio de las inequidades sociales es de primordial importancia para la salud pública y su análisis es un reto, toda vez que se ha reconocido que la pobreza y los problemas de salud son fenómenos interrelacionados, cuya asociación es causal y bidireccional. ${ }^{8}$

El estudio de las IS es un campo novedoso del conocimiento, caracterizado por un cuerpo teórico en construcción, que se ha venido fortaleciendo desde la segunda mitad del siglo XX, con importantes avances. Un ejemplo pionero se dio en Gran Bretaña en 1980, con la publicación del informe Black, ${ }^{3}$ que marcó un hito en la comprensión de cómo las condiciones sociales se transforman en inequidades en salud entre ricos y pobres y, evidenció la necesidad de intervenir en educación, vivienda y bienestar social, además de mejorar los servicios de salud.

Después, un grupo de investigadores identificó IS de alto impacto en salud en Europa. Dichos investigadores mostraron que en aquellos países y regiones donde los recursos económicos y sociales están mejor distribuidos, hay mejores niveles de salud. ${ }^{9}$ En consecuencia, los autores recomendaron desarrollar programas tendientes a disminuir las IS, más que a reducir la pobreza y concluyeron que la gobernanza, la distribución de ingresos y el trabajo, más que las condiciones individuales, son los determinantes de la salud.
Mármot, con sus estudios, mostró que los determinantes no son individuales y que las condiciones de salud y la mortalidad tienen gradientes sociales. Asimismo, dedujo que por encima de los bienes materiales están la autonomía, las oportunidades para cumplir compromisos sociales y la participación en la toma de decisiones, como elementos claves para la salud, el bienestar y la longevidad de las personas. Mármot definió los determinantes sociales como las circunstancias en las cuales nace, vive, crece, trabaja y envejece la gente mientras que la inequitativa distribución de poder, dinero y recursos, tejen esas circunstancias. ${ }^{10}$ En un reporte reciente se llegó a la conclusión que quienes viven en los lugares más deprimidos, pasarán 17 años más con discapacidades y morirán siete años más temprano que quienes viven en vecindarios ricos. Una de las principales recomendaciones es dar a todos los niños el mejor inicio en la vida, ${ }^{11}$ lo cual amerita una buena atención en salud materna.

Ahora bien, la OMS, preocupada por las IS, nombró una comisión de expertos en 2004 para revisar el tema, sistematizar los conocimientos y elaborar propuestas para reducir las inequidades. ${ }^{7}$ En 2008 se publicó el informe que definió como valores principales la equidad, los derechos humanos y la distribución del poder; entendido éste no sólo como poder de coerción sino como poder para la transformación. Además, propuso un marco para el estudio de las IS, que incluye los determinantes estructurales: contexto social, económico y político; la gobernanza, la política macroeconómica; las políticas sociales de empleo, vivienda y distribución de tierra; las políticas públicas de educación, salud y protección social; así como la cultura y los valores. Los sistemas de salud fueron considerados como determinantes intermedios de la salud de una población. ${ }^{4}$

Esta comisión de determinantes consideró tres perspectivas teóricas que intentan explicar los mecanismos por los cuales las condiciones sociales afectan la salud: la teoría psicosocial, la teoría de la producción social de la enfermedad y la teoría 
ecosocial. La primera argumenta que en sociedades muy desiguales, la autopercepción del lugar que se ocupa en la jerarquía social genera estrés, sentimientos de minusvalía y autodesprecio en las personas, lo cual altera su sistema neuroendocrino y origina enfermedad. La teoría de la producción social de la enfermedad hace énfasis en que la falta de recursos y la prioridad propia del capitalismo de acumular riqueza, poder y prestigio, lleva al ser humano a sacrificar el bienestar y propicia la enfermedad. Por su parte, la teoría ecosocial toma elementos de las dos anteriores y plantea que la "incorporación" de todos los factores, desde la biología, las condiciones sociales y materiales, en diferentes niveles, instaura patrones de salud y enfermedad en la población. ${ }^{12}$

En América Latina, el interés por los determinantes sociales inició con los trabajos de Salvador Allende, quien señaló que el principal condicionante de la salud es el nivel socioeconómico. ${ }^{13}$ Después, se han destacado científicos que han dinamizado el pensamiento sobre los determinantes sociales en salud, como Edmundo Granda, Jorge Breilh, Asa Cristina Laurell y Almeida-Filho; con sus trabajos en salud colectiva. ${ }^{14}$ Además, el Foro Social Mundial convoca a movilizarse contra la exclusión, la inequidad, la discriminación social y el daño a la naturaleza. Asimismo, defiende la idea que otro mundo es posible, más justo, solidario y equilibrado. ${ }^{15}$

\section{Políticas de atención materna e IS}

La atención materna y su importancia en la salud pública ha llevado a organismos internacionales, tales como la Organización de las Naciones Unidas (ONU), la OMS y la Organización Panamericana de la Salud (OPS), a orientar las políticas públicas en favor de la maternidad segura. Dan cuenta de ello la Estrategia de Atención Primaria (APS), promulgada en $1978^{16}$ y renovada en el $2005,{ }^{17}$ la Conferencia Internacional sobre Maternidad sin Riesgo de 1987, ${ }^{18}$ la XXIII Conferencia Sanitaria Panamericana, llevada a cabo en $1993,{ }^{19}$ los Objetivos de Desarrollo del Milenio (ODM) ${ }^{20}$ acordados en el año 2000 y el Informe sobre la Salud en el Mundo, publicado en el 2005 por la OMS, ${ }^{5}$ entre otras. Todas estas iniciativas cuentan con un componente central dirigido a disminuir las IS en la provisión de servicios de salud materna, tal como se muestra a continuación.

La Carta de Constitución de la OMS establece el goce del grado máximo de salud como derecho fundamental y consagra como contenidos del derecho a la salud: la lucha contra la desigualdad, el sano desarrollo de la infancia y la promoción y protección de la salud. ${ }^{21}$ Esta declaración da pie al reconocimiento del derecho de toda mujer a la salud materna en condiciones de equidad. Como confirmación, la Declaración Universal de los Derechos Humanos, en 1946, dejó en firme que tanto la maternidad como la infancia son etapas en las cuales las personas tienen derecho a cuidados y asistencia especiales. $^{2}$

También la prioridad dada a la salud materna fue explícita en el Pacto Internacional por Derechos Económicos, Sociales y Culturales firmado por la ONU. Con respecto a la igualdad y el derecho a la salud de las mujeres, el pacto enfatiza en la necesidad de educación e información, en particular en temas concernientes a la salud sexual y reproductiva. Exige además, que los servicios sean acordes con las necesidades de las mujeres y que estén disponibles en calidad y acceso comparables a los existentes para la atención de los hombres. ${ }^{22}$

En sintonía con lo anterior, la estrategia de APS, incluyó entre los cuidados mínimos, la educación, la atención materno-infantil y la planificación familiar. ${ }^{16}$ Estas medidas fueron acogidas en la Carta para la Promoción de la Salud de Ottawa (1986), ${ }^{23}$ en la iniciativa por una Maternidad sin Riesgo de Nairobi $(1987)^{18}$ y en la conferencia de Adelaida (Australia, 1988), en las que también se recomendó adaptar las prácticas obstétricas respetando las preferencias y necesidades de la gestante. ${ }^{24}$ Asimismo, en la Cuarta Conferencia Mundial sobre la Mujer (Beijing, 1995), se pidió igualdad, desarrollo y paz para todas las mujeres, con el reconocimiento de los 
derechos sexuales y reproductivos como derechos humanos. ${ }^{25}$ Luego en Bogotá, en 1992, se suscribió el compromiso de fortalecer la participación, eliminar las inequidades en contra de las mujeres y propiciar con ellas un diálogo de saberes. ${ }^{26}$

Ahora bien, en el año 2000, la ONU aprobó los ODM, 2000-2015, ${ }^{20}$ que incluyen la disminución de la mortalidad materna e infantil y las inequidades; objetivos directamente relacionados con la salud materna. Como complemento, en el 2003, la OMS instó a tomar en cuenta los principios de APS para apoyar las metas de los ODM. ${ }^{17}$ Estos compromisos están pendientes por cumplir y a pesar del contenido y la intencionalidad de las políticas, estas se desdibujan en la práctica. Por ejemplo, en Colombia, la Ley 100 de $1993^{27}$ fragmentó los servicios y llevó a que el CPN, que antes se prestaba cerca de las viviendas de las embarazadas, se trasladara a sitios distantes, lo cual se convierte en una barrera para el acceso, aumenta los costos y las inequidades en la prestación de los servicios. ${ }^{28}$

\section{El control prenatal y las inequidades en salud materna}

El control prenatal consiste en un programa de consultas dirigidas a la embarazada, que inician en el primer trimestre de gestación hasta terminar el embarazo, ${ }^{29}$ con lo cual se espera que cada mujer asista al menos a seis consultas. El CPN incluye pruebas de detección precoz y manejo oportuno de factores de riesgo, así como educación y consejería para la embarazada y su familia.

Sin embargo, la evidencia muestra que en pocas ocasiones el CPN se cumple. Por ejemplo, un estudio informó que sólo el 32,8\% de las embarazadas habían iniciado el CPN en las primeras 12 semanas, con un promedio de consultas de $4,6 .{ }^{30}$ Otro estudio reportó que sólo el 69\% de gestantes habían asistido al menos a una consulta durante el embarazo. ${ }^{31}$ Por último, en un trabajo con adolescentes, se encontró una mediana de 4 consultas durante la gestación y una mediana de 12 semanas de edad gestacional al inicio del CPN. ${ }^{32}$ Estos datos muestran que sólo en la mitad de las embarazadas el inicio del CPN fue oportuno y que el número de consultas en el 50\% de los casos restantes fue inferior al esperado.

En países desarrollados, por ejemplo Canadá, España o Australia, la razón de mortalidad materna (MM) para el 2004 fue de 5 por 100.000 nacidos vivos (NV) mientras en Afganistán fue de 1900 por 100.000 NV y en Sierra Leona 2000 por 100.000 NV. En Centroamérica, Haití fue el país con la razón más alta: 680 por 100.000 NV y Costa Rica tuvo la más baja: 25 por 100.000 NV. En América Latina, para el año de 1990, la razón de MM fue de 190 por 100.000 NV. Bolivia presentó la razón más alta: 420 por $100.000 \mathrm{NV}$ y Uruguay: 20 por $100.000 \mathrm{NV}$, la más baja. ${ }^{33}$ Esto demuestra las grandes inequidades entre países.

Una revisión sistemática de los estudios efectuados en 23 países en vías de desarrollo puso en evidencia inequidades en el uso de los servicios de salud materna. Identificó factores de la madre, como edad, educación, seguro médico; factores de riesgo clínicos; factores relacionados con la oferta de atención, como la disponibilidad de consultorios o la distancia de la vivienda; y otros, como la calidad de la atención. También hallaron brechas relacionadas con la financiación, la organización de la atención, así como aspectos sociales y culturales. Una de las recomendaciones fue el llamado a investigar y evaluar las IS en cada contexto específico. ${ }^{34}$

Otra revisión de estudios del Reino Unido mostró que los factores sociales están asociados tanto al inicio tardío del CPN, como al menor número de consultas. Hubo IS que afectaron a mujeres jóvenes, solteras, de raza no blanca, de ingresos bajos, alta paridad, bajo nivel educativo, bajo nivel socioeconómico y sin afiliación a la seguridad social. Las mujeres con baja asistencia al CPN también mostraron mayor riesgo de resultados adversos de su gestación. ${ }^{35}$

Otro estudio europeo encontró que las gestantes de clase alta realizaron mejor cuidado y control del embarazo, con más de doce visitas, más de tres ecografías, e incluso con excesiva medicalización. 
Por su parte, las gestantes de clase baja hicieron menos de seis visitas, la primera después del primer trimestre de embarazo, tuvieron menor posibilidad de realizar exámenes, tomar ácido fólico, planificar el embarazo y dejar de fumar. ${ }^{36}$

Ahora bien, en Bangladesh se hallaron inequidades en el uso de los servicios de salud materna en función de los quintiles de riqueza así: una oportunidad relativa (OR) de 2,51, (IC95\% 1,68$3,76)$ para atención por personal calificado, de 2,58, (IC95\%: 1,28-5,19) para conseguir cesárea y de 1,53 (IC95\%: 1,05-2,25) para uso de servicios de atención posparto, estuvieron asociadas al quintil más alto. Fueron barreras de acceso, la mayor distancia entre la vivienda y la unidad de salud, la residencia en el área rural y el bajo nivel educativo. ${ }^{37}$

En América Latina, los estudios sobre IS son escasos, no obstante que la región presenta contrastes: por un lado ha sido pionera en el desarrollo de la medicina social y por otro, cuenta con la peor distribución de los ingresos en el mundo. ${ }^{38}$

En un estudio realizado en Guatemala, se encontró un uso diferencial de los servicios según la etnia, con un gradiente entre las mujeres indígenas y las no indígenas. Las primeras buscan atención con parteras tradicionales más que con personal calificado, ya que con ellas tienen fácil acceso, mayor confianza y mejor comunicación. ${ }^{39}$

En Bolivia, se hallaron inequidades en la cobertura del CPN: 79\% del área urbana y 38\% rural; 60\% de gestantes hacen mínimo cuatro CPN en el área urbana y sólo 30\% rural. Según la etnia, la mitad de hispanoparlantes tuvieron CPN adecuado, mientras que este fue apenas de 25\% entre las indígenas. En cuanto al nivel educativo, las mujeres sin escolaridad obtuvieron un CPN adecuado en 35\% de los casos, 32\% quienes tenían primaria, 62\% aquellas con secundaria y $84 \%$ las mujeres con educación superior. Además el 10\% de quienes tuvieron CPN adecuado corresponden al quintil inferior de ingresos y el $30 \%$ al quintil superior. ${ }^{40}$

En Colombia, los reportes sobre IS consisten en análisis ssecundarios. Por ejemplo, datos de la Encuesta de Demografía y Salud del año 2005 muestran que las embarazadas que viven en la Región del Pacífico tienen menos probabilidades de asistir al CPN. Las mujeres con más ingresos tienen mejor oportunidad de obtener CPN sin pagos adicionales; asimismo, un alto nivel educativo y tener pareja estable fueron asociadas con mejor asistencia al $\mathrm{CPN}^{41}$

Un estudio similar informó disminución de las inequidades entre los años 1990 y 2005, sustentado por el aumento de la atención médica del parto del $72,8 \%$ al $89,5 \%$, y de la atención prenatal adecuada (al menos 4 CPN) del 70,7\% al 84,3\%, principalmente en mujeres pobres del área urbana. Sin embargo, aún las mujeres con ingresos altos obtienen CPN y atención del parto adecuado 40\% más que las pobres. ${ }^{42}$

Una investigación con adolescentes embarazadas mostró las IS según escolaridad, dado que el 51\% de ellas no tenían ningún grado de educación, el 38\% habían realizado la primaria y el 11\% la secundaria o más. También se identificó un gradiente por quintil de riqueza, pues el 30\% de ellas provenía del quintil más pobre y sólo el 10\% del quintil más rico. Además, el 80\% de las madres adolescentes de estrato bajo no se reintegra al sistema escolar, entonces se interrumpe la educación y se refuerza el círculo de la pobreza. ${ }^{43}$ Ahora bien, el bajo peso al nacer fue asociado al nivel socioeconómico bajo de las madres, como un reflejo de las IS en la salud materna. ${ }^{44}$

Estas IS se dan en Colombia a pesar de que las gestantes están amparadas por la Constitución Política (Artículo 43) y la Política Nacional de Salud Sexual y Reproductiva, que contempla la búsqueda de equidad de género, justicia social, igualdad de trato, de oportunidades y el empoderamiento de las mujeres. ${ }^{45}$

\section{Necesidades de evidencia en Colombia}

Se requiere caracterizar las IS que afectan el acceso y el uso de los servicios de atención materna, tanto en el país en general, como en las diferentes regiones, 
acogiendo las recomendaciones de estudios previos, en el sentido de realizar investigación en cada contexto, dada la diversidad de inequidades. ${ }^{34}$ Los resultados permitirán perfilar el problema, colocarlo en la agenda social y aportar datos que sustenten intervenciones y sirvan de base para realizar seguimiento.

Además, esta generación de conocimiento servirá para orientar los servicios de salud en acciones tendientes a lograr equidad, para formar investigadores en el tema, mejorar la producción científica en lengua castellana, validar las teorías y promover el empoderamiento de las mujeres sobre sus derechos en salud.

\section{Conclusiones y recomendaciones}

Existen IS en la provisión, acceso y uso de los servicios de atención materna, a pesar de las iniciativas de los organismos internacionales de promover la equidad mediante la atención universal con calidad y justicia para todas las mujeres. Esta reflexión refuerza el concepto de que las inequidades son desigualdades evitables, injustas e inadmisibles, dado que podrían ser superadas con la puesta en práctica de las políticas públicas incluyentes y equitativas, promulgadas por los organismos internacionales. Se requieren estudios en el contexto nacional para orientar las intervenciones, probar las teorías, mejorar el conocimiento, formar el talento humano y contribuir a la disminución de las IS en el cuidado materno; más aún, cuando la brecha en la salud materna, según las condiciones sociales, es cada vez mayor.

\section{REFERENCIAS}

1. Whitehead M. The concepts and principles of equity and health. Int J Health Serv 1992;22:430-45.

2. Organización de las Naciones Unidas. Declaración Universal de los Derechos Humanos. 1948. Visitado 2010 Mar 5. Disponible en: http://www.un.org/es/ documents/udhr/index.shtml

3. Department of Health and Social Security. Inequalities in health: report of research working group. London: DHSS; 1980. (Black Report).
4. Solar O, Irwin A. A conceptual framework for action on the social determinants on health. Discussion paper for the Commission on social determinants of health. Geneva: WHO; 2007.

5. Organización Mundial de la Salud. Informe sobre la salud en el mundo 2005. Cada madre y cada niño contarán. Ginebra, Suiza; 2005.

6. República de Colombia. Asociación Probienestar de la Familia Colombiana (PROFAMILIA). Salud sexual y reproductiva. Informe de la Encuesta Nacional de Demografía y Salud 2005. Visitado 2010 Mar 10. Disponible en: http://www.profamilia.org.co/ encuestas/index.htm

7. Irwin A, Valentine N, Brown C, Loewenson R, Solar O, Brown H, et al. The Commission on Social Determinants of Health: Tackling the Social Roots of Health Inequities. PloS Medicine 2006;3(6). Visitado 2010 Mar 5. Disponible en: http:/www.plosmedicine.org/article/ info\%3Adoi\%2F10.1371\%2Fjournal.pmed.0030106

8. Wastaff A. Pobreza y desigualdades en el sector salud. Rev Panam Salud Pública 2002;11:316-26.

9. Navarro V. The political and social contexts of health. Amityville, New York: Baywood Publishing Company, INC.; 2004.

10. Marmot M, Friel S. Global Health equity: evidence for action on the social determinants of health. J Epidemiol Community Health 2008;62:1095-7.

11. Marmot M. Fair society, healthy lives. The Marmot Review. Strategic Review of health inequalities in England post-2010. Visitado 2010 Mar 12. Disponible en: http://www.marmotreview.org

12. Krieger N. Proximal, distal and the political of causation: what's level got to do with it? Am J Public Health 2008;98:221-9.

13. Allende S. La realidad médico-social chilena. Ministerio de Salud de Chile, Santiago de Chile; 1939.

14. Hernández LJ. Qué critica la epidemiología crítica. Una aproximación a la mirada de Naomar Almeida Filho. Bol Observ Salud 2009;2:18-28.

15. De Sousa-Santos B. Foro Social Mundial. Manual de uso. Visitado 2009 Abr 12. Disponible en: http://www.icariaeditorial.com/libros 2 . php?k $=3 \& b=2 \& i d=734 \& b 2=$ foro

16. Organización Mundial de la Salud. Declaración de Alma-Ata 1978. Salud para todos. Visitado 2010 Ene 24. Disponible en: http://www.ops.org.bo/ alma_atta/declaracion.pdf 
17. Organización Mundial de la Salud. Renovando la atención primaria de la salud en las Américas. Washington DC, 2005. Visitado 2010 Feb 28. Disponible en: www.paho.org/Spanish/AD/THS/OS/ PHC_brochure_spa.pdf

18. Banco Mundial, Organización Mundial de la Salud. La Iniciativa por una Maternidad sin riesgo. Nairobi, 1987. Visitado 2010 Mar 30. Disponible en: http://www.icmer.org/RHO/html/sm_overview. htm\# sminitiative

19. Organización Panamericana de la Salud, Organización Mundial de la Salud. 25 a Conferencia Sanitaria Panamericana. 50 Sesión del Comité Regional.Washington DC; 1998. Visitado 2010 Mar 20. Disponible en: http://www.paho.org/Spanish/gov/csp/csp25_8.pdf

20. Reunión de Alto Nivel, Naciones Unidas, Nueva York, 25 de Septiembre de 2008. Objetivos de Desarrollo del Milenio. Visitado 2008 Nov 15. Disponible en: http://www.un.org/spanish/millenniumgoals/pdf/ MDGOverviewSPANISH.pdf

21. Organización Mundial de la Salud. Constitución. Documentos básicos, suplemento de la $45^{\text {a }}$ edición. Visitado 2009 Ago 23. Disponible en: www.who.int/ governance/eb/who_constitution_sp.pdf

22. Organización de las Naciones Unidas. Consejo Económico y Social. Comité de Derechos Económicos, Sociales y Culturales. Ginebra, Suiza, 2002. Visitado 2009 Nov 12. Disponible en: http://www. cohre.org/store/attachments/RTWP\%20-20Observacion\%20General\%20No.\%2015.pdf

23. Organización Mundial de la Salud, Organización Panamericana de la Salud. Carta de Ottawa para la Promoción de la Salud. Visitado 2010 Feb 25. Disponible: https://iehost.net/pdf/conf1.pdf

24. World Health Organization. Adelaide Recommendations on Healthy Public Policy. Second International Conference on Health Promotion, Adelaide, South Australia, 5-9 April 1988. Visitado 2009 Sep 10. Disponible en http://www.who.int/healthpromotion/ conferences/previous/adelaide/en/index.html

25. Organización de las Naciones Unidas. Cuarta Conferencia Mundial sobre la Mujer. Beijing, China, Septiembre de 1995. Visitado 2009 Sep 20. Disponible en: http://www.un.org/spanish/conferences/ mujer.htm
26. OPS/OMS, República de Colombia/Ministerio de Salud, 1992. Conferencia Internacional de Promoción de la Salud. Santafé de Bogotá, noviembre de 1992. Visitado 2010 Sep 10. Disponible en: http://www.paho.org/ Spanish/AD/SDE/HS/MTC_DeclaracionBogota.pdf

27. República de Colombia, Ministerio de Salud. Ley 100 de 1993. Sistema General de Seguridad Social en Salud. 1993. Visitado 2008 Oct 20. Disponible en http://www. minproteccionsocial.gov.co/vbecontent/librar y/documents/DocNewsNo17981DocumentNo10845.pdf

28. Hernández-Álvarez ME. El concepto de equidad y el debate sobre lo justo en salud. Rev Salud Pública 2008;10:72-82.

29. República de Colombia, Ministerio de Salud. Norma técnica para la detección temprana de las alteraciones del embarazo. (Resolución 412 del 2000). Visitado 2008 Oct 20. Disponible en http://www.minproteccionsocial.gov.co/VBeContent/library/documents/ DocNewsNo369511.pdf

30. Ruiz M, Cristancho LP, Ramírez C. Características del programa de control prenatal en instituciones de salud pública de Bucaramanga. MÉDICAS UIS 2001; 15:159-68.

31. Observatorio de Salud Pública de Santander. Sala de situación: mortalidad materna y perinatal en Santander. Bucaramanga, junio 30 de 2005. Visitado 2008 Nov 22. Disponible en http://www.observatorio.saludsantander. gov.co/foros/Documento\%20Mortalidad\%20Materna\%20Perinatal.pdf

32. Ortiz R, Anaya NI, Sepúlveda C, Torres SJ, Camacho P. Caracterización de las adolescentes gestantes de Bucaramanga, Colombia. Un estudio transversal. MedUnab 2005;8:71-7.

33. Organización Mundial de la Salud. Informe sobre la salud del mundo 2004. Cambiemos el rumbo de la historia, anexo estadístico. Visitado 2009 Nov 20. Disponible en: www.who.int/whr/2004/annex/es/index.html

34. Say L, Raine R. A systematic review of inequalities in the use of maternal health care in developing countries: examining the scale of the problem and the importance of context. Bull World Health Organ 2007;85:812-9.

35. Rowe RE, Garcia J. Social class, ethnicity and attendance for antenatal care in the United Kingdom: a systematic review. J Public Health Med 2003;25:113-9. 
36. Cano-Serral G, Rodríguez-Sánz M, Borrel C, Pérez MM, Salvador J. Desigualdades socioeconómicas relacionadas con el cuidado y el control del embarazo. Gac Sanit 2006;20:25-30.

37. Anwar I, Sami M, Akhtar N, Chowdhury ME, Salma U, Rahman M, et al. Inequity in maternal health-care service: evidence from home-base skilled-birthattendant programs in Bangladesh. Bulletin of the World Health Organization 2008;86:252-59.

38. Naciones Unidas. Comisión Económica para América Latina y el Caribe (CEPAL). Cumbre de las Américas 1994-2009. Indicadores seleccionados. Visitado 2009 Jun 3. Disponible en: http://www.cepal.org/cgi-bin/ getProd.asp?xml =/publicaciones/xml/5/35755/ $\mathrm{P} 35755 . \mathrm{xml} \& \mathrm{xsl}=/ \mathrm{tpl} / \mathrm{p} 9 \mathrm{f} . \mathrm{xsl} \& \mathrm{base}=/ \mathrm{tpl} / \mathrm{top}$ bottom.xsl

39. Glei DA, Golgman N, Rodríguez G. Utilization of care during pregnancy in rural Guatemala: does obstetrical need matter? Soc Sci Med 2000;57:2447-63.

40. Narváez-Guzmán R. Inequidades en el estado de salud, acceso y uso de los servicios de salud, en Bolivia 1998.
Unidad de análisis de políticas sociales y económicas, UDAPE. La Paz, Bolivia; 2002.

41. Vecino-OrtizAI. Determinants of demands for antenatal care in Colombia. Health Policy 2008;86:363-72.

42. Flórez CE, Soto VE, Acosta OL, Karl C, Misas JD, Forero N, et al. Avances y desafíos de la equidad en el sistema de salud colombiano. Fundación Corona, DNP, Universidad de Los Andes, Universidad del Rosario. Bogotá, Colombia; 2007.

43. Flórez CE, Soto VE. Fecundidad adolescente y desigualdad en Colombia y América Latina y el Caribe. CEDE, Universidad de los Andes, Bogotá Colombia. p 25.

44. Gaitán H, Delgado M. El bajo peso al nacer: otro ejemplo de inequidad en Colombia. Rev Colomb Obstet Ginecol 2009;60:121-3.

45. República de Colombia. Ministerio de la Protección Social. Política Nacional de Salud Sexual y Reproductiva. Visitado 2009 May 26. Disponible en: http://www. minproteccionsocial.gov.co/VBeContent/newsdetail. asp $\mathrm{id}=15132$ \&idcompany $=3$

Conflicto de intereses: ninguno declarado.

Fuente de financiación: Actualmente, Flor de María Cáceres recibe comisión de estudios por parte de la Universidad Industrial de Santander (UIS). Este artículo es producto de la revisión teórica realizada para sustentar la propuesta de Tesis Doctoral ante la Facultad Nacional de Salud Pública de la Universidad de Antioquia. 